\title{
Resistive cooling circuits for charged particle traps using crystal resonators
}

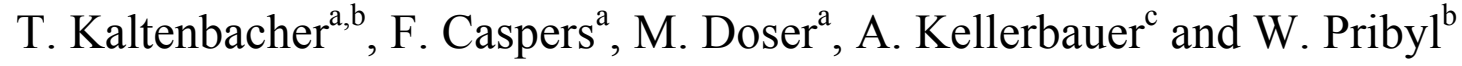 \\ ${ }^{a}$ Physics and Accelerator Departments, CERN, 1211 Geneva 23, Switzerland \\ ${ }^{b}$ Institute of Electronics, Graz University of Technology, Inffeldgasse 12, 8010 Graz, Austria \\ ${ }^{c}$ Max Planck Institute for Nuclear Physics, Saupfercheckweg 1, 69117 Heidelberg, Germany
}

\begin{abstract}
The paper addresses a novel method to couple a signal from charged particles in a Penning trap to a high Q resonant circuit using a crystal resonator. Traditionally the trap capacity is converted into a resonator by means of an inductance. The tuned circuit's Q factor is directly linked to the input impedance "seen" by the trapped particles at resonance frequency. This parallel resonance impedance is a measure of the efficiency of resistive cooling and thus it should be optimized. We propose here a commercially available crystal resonator since it exhibits a very high $\mathrm{Q}$ value and a parallel resonance impedance of several $\mathrm{M} \Omega$. The possibility to tune the parallel resonance frequency of the quartz results in filter behavior that allows covering a range of some tens of its 3 -dB bandwidth by means of tuning.
\end{abstract}

Keywords: Penning Trap; Tank circuit; Crystal resonator; Resistive cooling.

PACS: 37.10.Rs (Ion cooling); 37.10.Ty (Ion trapping); 52.27.Jt (Nonneutral plasmas); 52.70.-m (Plasma diagnostic techniques and instrumentation).

\section{Introduction}

In many experiments using ion traps, the kinetic energy of confined particles must first be reduced in order to perform high precision measurements [1]. Laser cooling is widely used and very effective, but it is limited to specific ions [1]. Methods which can theoretically be applied for all ions are active-feedback cooling, collisional cooling, RF cooling, evaporative cooling and resistive cooling (occasionally called electronic cooling) [2, 3]. In resistive cooling, the trap electrodes are connected to an external circuit to dissipate energy from the ion through induced currents [1]. In other words, the kinetic energy of the confined particles is damped by $i^{2} R$ losses in a resistive circuit. Grossly speaking, the resistor absorbs the particles' energy and is heated up in the process. Since the resistor has a specific physical temperature, it generates Johnson noise that in turn stochastically drives the trapped particles [4]. In the absence of other heating sources, a thermal equilibrium between the particles and the resistive circuit is ultimately established.

A single ion confined in an ideal Penning trap, consisting of a solenoidal magnetic field and a threedimensional electric quadrupole field, performs three fundamental particle motions, namely axial $\left(\omega_{z}\right)$, cyclotron $\left(\omega_{c}\right)$ and magnetron $\left(\omega_{m}\right)$ oscillations [4]. The axial motion is the oscillation parallel to the magnetic field $(z$ direction) and its frequency is determined by the electrical field only [4]. The axial frequency of a single particle with mass $m$ and charge $q$ is given by

$$
\omega_{z}=2 \pi v_{z}=\sqrt{\frac{q V_{0}}{m d^{2}}},
$$

where $V_{0}$ is the electrostatic potential applied to the electrode and $d$ is a characteristic trap parameter [4]. A large collection of ions in a Penning trap arranges into a plasma whose spatial extent is large compared with the pene- 


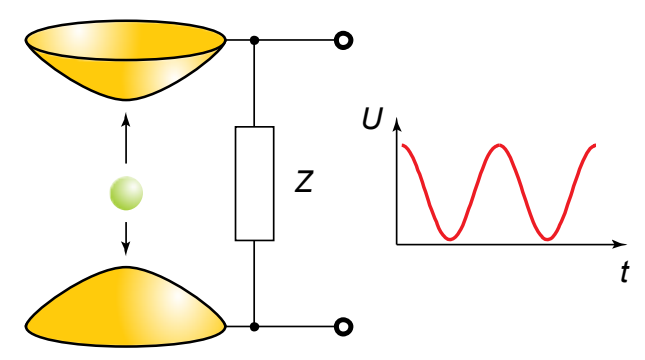

Fig. 1: Principle of resistive cooling of trapped ions.

tration depth of a static electric field, called the Debye length. In thermal equilibrium, the motions of the particles become strongly correlated and they form an ellipsoid which performs a rigid rotation about the trap axis [5]. Along the trap axis, the ellipsoid performs collective oscillations which deform or displace the ion cloud. In the low-temperature limit, the plasma oscillations can be described by a cold-fluid theory [6] and their respective frequencies can be determined analytically. The lowest-order axial mode, also called the 'bounce' mode, corresponds to a periodic axial displacement of the entire plasma. Its oscillation frequency $\omega_{1}=\omega_{z}$ is identical to the axial frequency of a single ion in a Penning trap.

To estimate the cooling time of the axial motion, a simple single-particle model is used, where the particle is harmonically confined between two infinite capacitor plates [7]. According to this model, the energy is damped with a time constant $\tau$ given by

$$
\tau=\frac{4 m z_{0}^{2}}{q^{2} R}
$$

where $R$ stands for the real part of the impedance of the attached external circuit. The induced current is given by

$$
i=\frac{q v_{z}}{2 z_{0}}
$$

where $2 z_{0}$ is the separation of the capacitor plates and $v_{z}$ is the particles' velocity in axial direction.

From Eq. (2) one can easily deduce that light, highly charged particles are efficiently cooled. The cooling rate can be further improved by developing a high resistance in the external circuit. In general, the external circuit includes a low-noise amplifier to couple the induced current signal to room temperature, and thus enable stored particle and plasma diagnostics. Traditionally, the impedance $Z$ shown in Fig. 1 is implemented as an inductance $L$ to tune out the parasitic capacitance $C$ of the electrodes [4]. This inductance usually consists of a discrete coil made of copper or superconducting wire. At the resonance frequency $\omega_{r}=(L C)^{-1 / 2}$ of a parallel LC circuit, the real part $R$ of the impedance is maximal and is given by

$$
R=\omega_{r} L Q
$$

Therefore, the quality factor $Q$ of the tuned circuit has to be as large as possible to guarantee efficient resistive cooling. Furthermore, also the coil's inductance $L$ is made as large as possible to increase the resistance in resonance. However, this only holds when the capacitance of the circuit can be freely chosen. Usually the capacitance is given by the Penning trap itself and all parasitic capacitances in the system. A large inductance in turn means that the coil has to have a certain size, since the inductance is basically given by its dimensions and the number of turns. Moreover, superconducting coils require careful shielding from magnetic fields as well as care in the design of the interface between the superconducting and resistive wire [8]. 


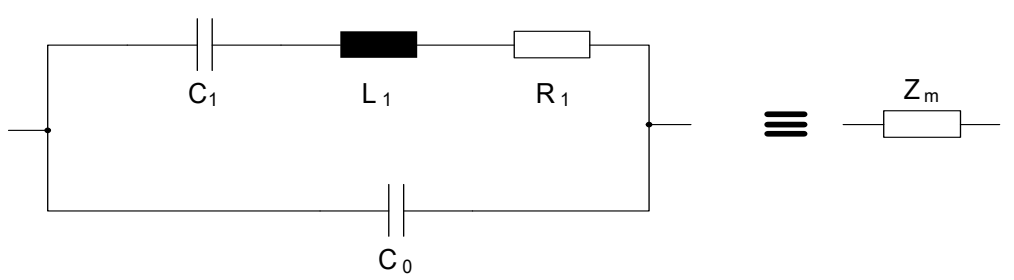

Fig. 2: Equivalent circuit for crystal resonator and simplified impedance.

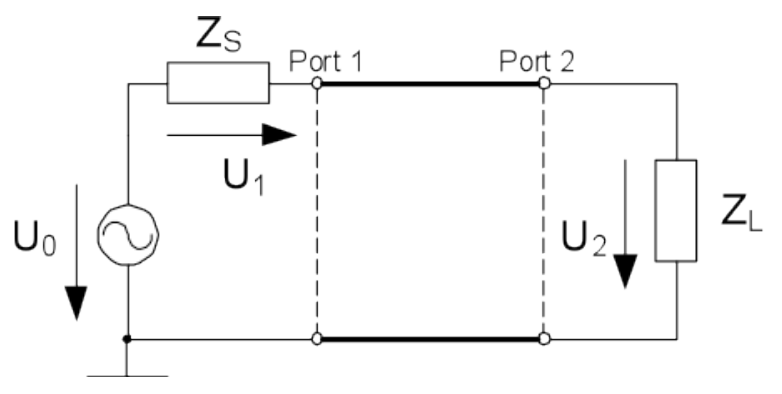

Fig. 3: Vector Network Analyzer measurement setup without crystal resonator.

\section{Resistive Cooling with a crystal resonator}

We propose and have investigated the use of a crystal resonator in parallel resonance instead of a coil. This setup provides several advantages:

- The crystal's operation is not negatively affected by the magnetic field of the Penning trap;

- $\quad$ The $Q$ factors of crystal resonators are very high, resulting in a very high impedance in resonance;

- A crystal resonator circuit is mechanically smaller than a superconducting coil with its shielding.

Quartz crystals are used in electronic circuits to provide very stable oscillation frequencies. Figure 2 shows the electrical equivalent circuit of a quartz crystal resonator. Its series and parallel structure leads to two resonances with the series and parallel resonance frequencies $\omega_{s}$ and $\omega_{p}$ :

$$
\begin{gathered}
\omega_{s}=\frac{1}{\sqrt{L_{1} C_{1}}} \\
\omega_{p}=\frac{1}{\sqrt{L_{1} C_{1}}} \sqrt{1+\frac{C_{1}}{C_{0}}}=\omega_{s} \sqrt{1+\frac{C_{1}}{C_{0}}} .
\end{gathered}
$$

The parallel resonance is also called anti-resonance and occurs when the parallel combination of $C_{1}$ and $C_{0}$ tunes out the inductance $L_{1}$. The capacitance of the crystal holder and parasitic capacitances is denoted by $C_{0}$. The resistance $R_{1}$ stands for electrical losses due to damping, and $L_{1}$ and $C_{1}$ are related to the mechanical properties of the quartz [9]. The equivalent circuit model, so called Butterworth Van-Dyke model, is valid for the single series, parallel resonance combination at frequencies near resonance [10]. Depending on the crystal geometry, a crystal oscillator may also oscillate at odd multiples of its series resonance frequency.

\section{Measurement setup}


To measure high impedances within a $50 \Omega$ system one has to use transmission measurements since the un-

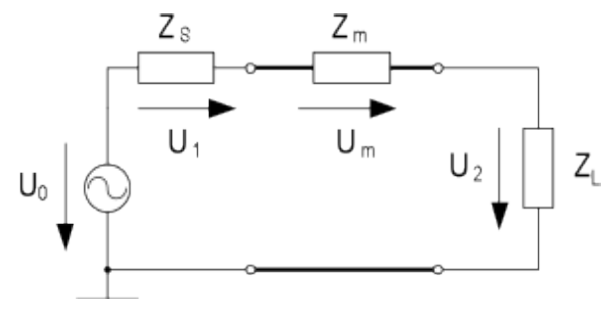

Fig. 4: Measurement setup including Vector Network Analyzer and crystal resonator.

known, high impedance cannot be measured in parallel. A sensitive spectrum analyzer with tracking generator

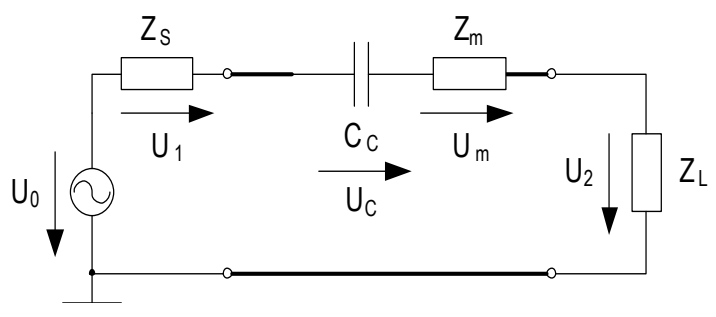

Fig. 5: Measurement setup including Vector Network Analyzer, coupling capacitance $C_{C}$ and crystal resonator.

could be used as well as a network analyzer. Our measurements were made with a vector network analyzer (VNA; Agilent 5071C). Figure 3 shows the schematic of the measurement setup without and Fig. 4 with the impedance to be measured $\left(Z_{\mathrm{m}}\right)$. The scatter parameter measured with the VNA is $S_{21}$ since in the transmission measurement the stimulus is applied to Port 1 and the signal is measured at Port 2. When using a VNA the measured quantity at Port 2 is complex, which in turn means that the signal's magnitude and phase are determined.

The measured voltage $U_{2}$ is given by

$$
U_{2}=U_{0} \frac{Z_{L}}{Z_{S}+Z_{L}}
$$

For $Z_{L}=Z_{S}$ it is equal to $U_{0} / 2$ and is called the reference voltage $U_{\text {ref, }}$ since it is used normalize the measured potential. The normalization of the measured quantity including the unknown impedance $Z_{m}$ is expressed by

$$
S_{21}=\frac{U_{2}}{U_{\text {ref }}}=\frac{2 Z_{L}}{Z_{S}+Z_{L}+Z_{m}} .
$$

Thus Eq. (8) gives the transmission scatter parameter $S_{21}$ which is measured with the VNA.

To simulate the electrical effects of a connected Penning trap, a coupling capacitance with $C_{C} \geq 10 \mathrm{pF}$ was introduced (see Fig. 5), which represents the electrode's capacitance as well as parasitic capacitances. In fact the load capacitances of the crystal resonators recommended by the suppliers were used as coupling capacitance. A capacitance in series with the quartz shifts the series resonance frequency $\omega_{s}$ toward the parallel resonance frequency:

$$
\omega_{s, \text { new }}=\frac{1}{\sqrt{L_{1} C_{1}}} \sqrt{1+\frac{C_{1}}{C_{0}+C_{C}}}=\omega_{s} \sqrt{1+\frac{C_{1}}{C_{0}+C_{C}}} .
$$


The parallel resonance frequency is only affected by capacitances in parallel with the quartz [9].

Tab. 1: Summary of the impedance amplitude $Z_{\text {res }}$ and the resulting quality factor $Q_{p}$ at parallel resonance $v_{p}=\omega_{p} /(2 \pi)$ of the measured crystal resonators at room temperature.

\begin{tabular}{|c|c|c|c|c|c|c|}
\hline \multirow[b]{3}{*}{$Z_{p}(\mathrm{k} \Omega)$} & \multicolumn{2}{|c|}{ AEL Crystals } & \multicolumn{2}{|c|}{ Euro Quartz } & \multicolumn{2}{|c|}{ KVG Crystal } \\
\hline & no $C_{C}$ & $C_{C}=33 \mathrm{pF}$ & no $C_{C}$ & $C_{C}=18 \mathrm{pF}$ & no $C_{C}$ & $C_{C}=22 \mathrm{pF}$ \\
\hline & 250 & 231 & 869 & 944 & 4,631 & 4,495 \\
\hline$v_{p}(\mathrm{MHz})$ & 20.0314 & 20.0312 & 20.0336 & 20.0334 & 19.4786 & 19.4780 \\
\hline$Q_{p}$ & 77,800 & 73,500 & 87,100 & 96,500 & 173,143 & 177,072 \\
\hline
\end{tabular}

\section{Frequency response}

First measurements with commercially available $20 \mathrm{MHz}$ crystals were carried out in order to determine the range of possible impedance values and to verify that the absolute value of the impedance at parallel resonance is large enough for efficient resistive cooling of trapped particles. Tab. 1 summarizes the measured values of three off-the-shelf crystals in order to show that there is no severe effect of a coupling capacitance $\mathrm{C}_{\mathrm{C}}$ on the impedance at parallel resonance and to compare their performance. The coupling capacitances were chosen according to the specifications given in the datasheets $[11,12,13]$. The $20 \mathrm{MHz}$ AEL Crystals and Euro Quartz resonators are housed in a hermetically sealed can with HC49 holder and nitrogen atmosphere [11, 12]. The 19.44MHz quartz resonator from KVG Quartz Crystal Technology has a HC43/U holder and is vacuum-sealed [13]. All three resonators oscillate in the fundamental mode. The observed shift of the parallel resonance frequency may be due to parasitic capacitances caused by the incorporation of the coupling capacitance into the circuit.

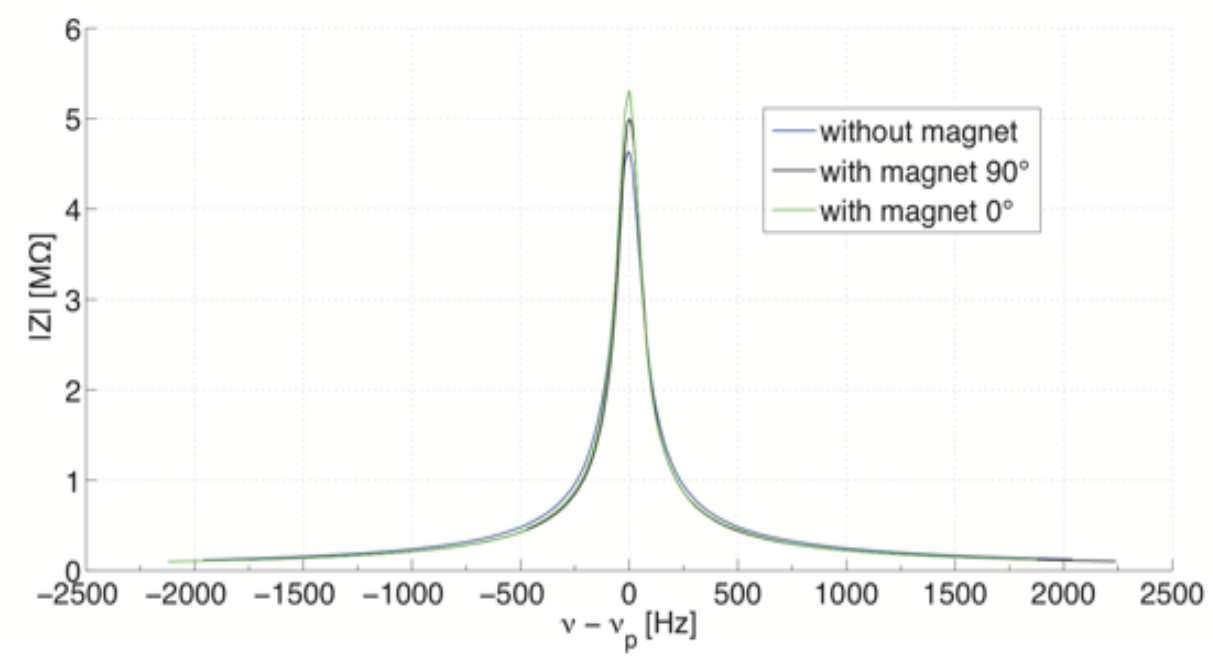

Fig. 7: Frequency response of KVG crystal at parallel resonance at room temperature with and without magnetic field. 


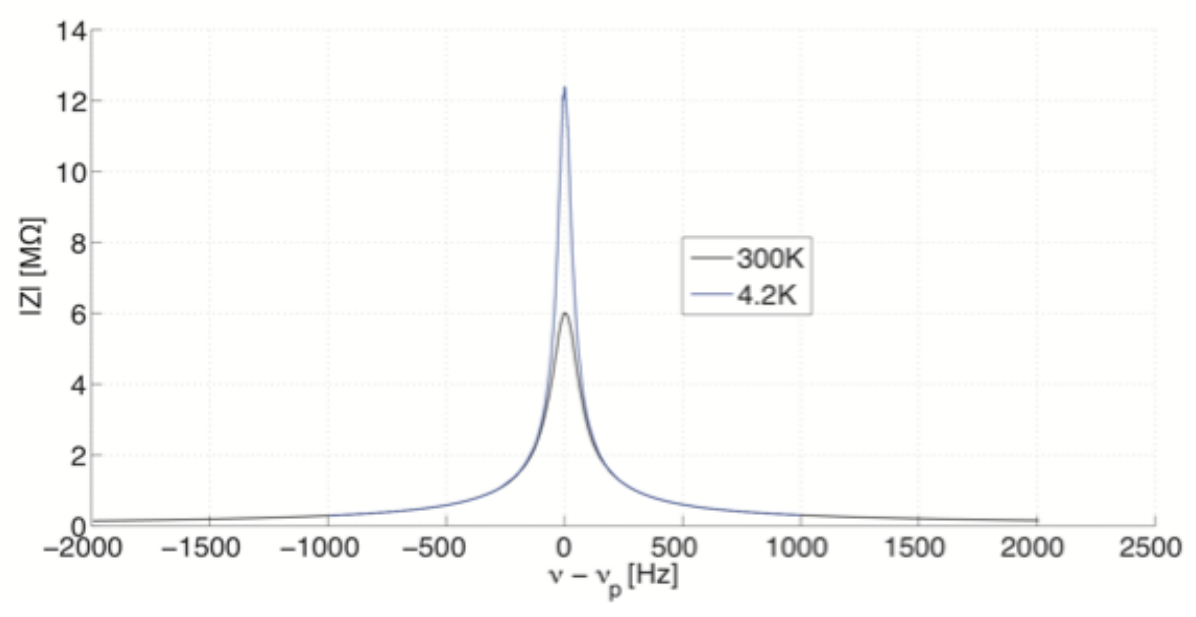

Fig. 8: Frequency response of KVG crystal at parallel resonance, at $300 \mathrm{~K}$ and $4.2 \mathrm{~K}$.

Since Penning traps are usually placed in cryogenic environments with a large DC magnetic field, further measurements were made to investigate the proper operation of the quartz under these conditions. In order to operate quartz crystals in a cryogenic environment, vacuum-sealed crystals must be used since gases like nitrogen used as atmosphere in the crystal housing would freeze out and could severely affect the crystal's function. This is the reason why measurements at $4 \mathrm{~K}$ were done with the KVG crystal only. The measurements shown in Fig. 6 were made at room temperature and in the absence of a magnetic field. Compared to the previously shown results, the KVG device is clearly superior with a higher impedance of about $4.5 \mathrm{M} \Omega$ at parallel resonance (see Tab. 1). This value is about four times larger than the highest value measured with the Euro Quartz device at room temperature. The parallel resonance frequency was found to be $v_{p}=19.4787 \mathrm{MHz}$ and the quality factor $Q_{p} \approx 173,100$ without coupling capacitor. The resonance frequency varied by less than $1 \mathrm{kHz}$ when the coupling capacitors were attached to the circuit, and there was no significant reduction of the impedance at $v_{p}$.

A large magnetic field was created by two permanent magnets (Webcraft) [14] which can develop a field magnitude of about $1 \mathrm{~T}$ close to the magnet. By attaching the magnets directly to the crystal's housing, the distance between the quartz and the magnet was minimized, thereby maximizing the magnetic field. No decisive effects were observed when the magnetic field was present from two different directions. Figure 7 shows the magnitude of the impedance when the magnetic field is perpendicular to the crystal disc ("with magnet $90^{\circ}$ ") and in parallel (“with magnet $0^{\circ}$ ") respectively. The cryogenic environment was realized with a Dewar filled with liquid helium.

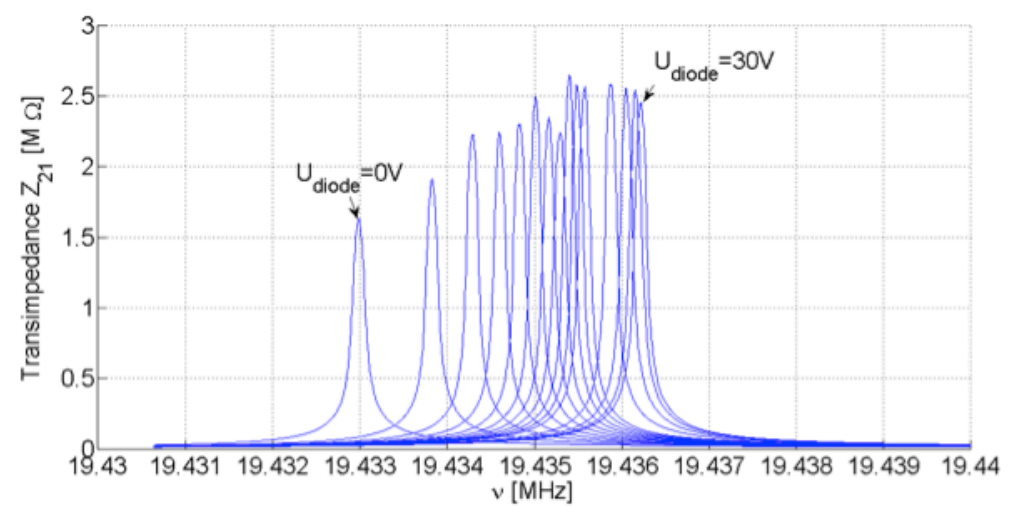

Fig. 10: Transimpedance for sweep of parallel resonance frequencies for different diode voltages at $4.2 \mathrm{~K}$. 


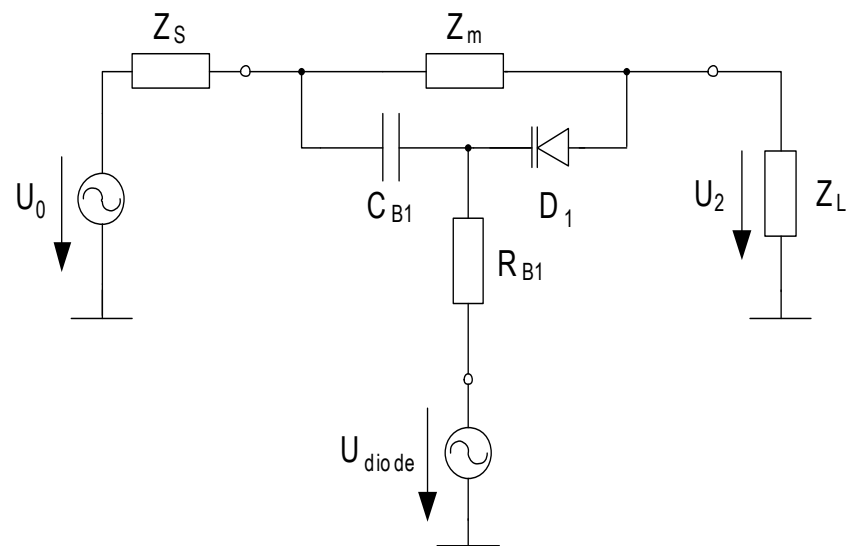

Fig. 9: Schematic for the circuit which allows to tune the parallel resonance frequency of the quartz resonator.

The cool-down from room temperature to liquid-helium temperature was carried out over about 15 min in order to minimize mechanical stress to the crystal. Figure 8 shows the result of the measurement at cryogenic temperature compared to the one at room temperature. As expected, there is an increase of the quality factor $Q_{p}$ and the resonance frequency is shifted toward lower frequencies [15]. $\mathrm{Q}_{\mathrm{p}}$ is increased by a factor of two. The measurement results show that the crystal resonator is fully functional in a cryogenic environment and in the presence of a strong magnetic field, as present in a Penning trap. Our results confirm earlier measurements presented in Refs. [16] and [17].

\section{Resonance frequency shift}

In a real Penning trap, ions may exhibit a range of resonance frequencies, depending on trap imperfections or their interactions with other trapped particles. It is thus desirable to be able to vary the resonance frequency and possibly chirp the parallel resonance of the crystal resonator over a range of frequencies. To shift the parallel resonance frequency of a quartz the schematic shown in Fig. 9 is used. The resistor $R_{B 1}$ is chosen as $1.5 \mathrm{M} \Omega$ to be large enough to not degrade the peak impedance at parallel resonance and to decouple the voltage source from the resonance circuit. The capacitance of the blocking capacitor $\mathrm{C}_{\mathrm{Bl}}$ must be larger than the minimum capacitance $C_{\text {diode }}$ of the diode $\mathrm{D}_{1}$ since $\mathrm{C}_{\mathrm{Bl}}$ and $\mathrm{C}_{\mathrm{D} 1}$ are in series and the lowest capacitance dominates. The diode $\mathrm{D}_{1}$ is a GaAs Schottky diode which has a capacitance of $C_{\text {diode }}=5.8 \mathrm{pF}$ at $U_{\text {diode }}=0 \mathrm{~V}$ and $C_{\text {diode }}=1.4 \mathrm{pF}$ at $U_{\text {diode }}=30 \mathrm{~V}$ and functions at cryogenic temperatures. The voltage-adjustable parallel capacitance is thus tunable from $C_{P}=$ $1.48 \mathrm{pF}$ to $0.82 \mathrm{pF}$. The result of the parallel resonance sweeping at liquid Helium temperature is shown in Figs. 10 and 11 . With this setup a tunability of the parallel resonance of more than $3 \mathrm{kHz}$ is realized.

The change of the parallel resonance frequency due to a parallel capacitance $C_{P}$ is given by

$$
\omega_{p}=\omega_{s} \sqrt{\frac{C_{0}+C_{1}+C_{P}}{C_{0}+C_{P}}} .
$$




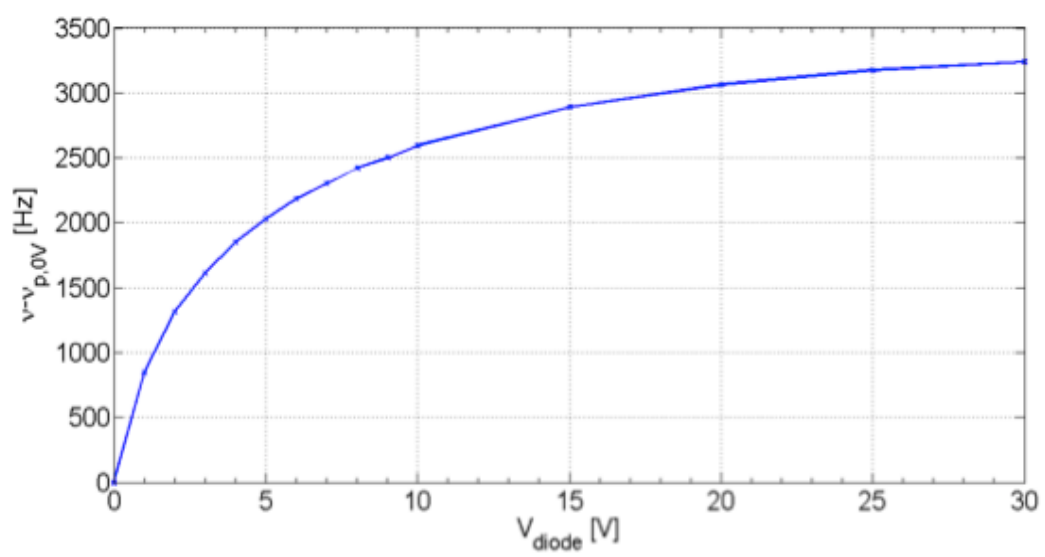

Fig. 11: Parallel resonance frequency $v_{p}$ shift normalized to the lowest $v_{p}$ at $0 \mathrm{~V}$ diode bias and at $4.2 \mathrm{~K}$.

The capacitance obtained by combining the blocking capacitance and the tuning capacitance of the diode in series is given by

$$
C_{P}=\frac{C_{\mathrm{Bl}} C_{\text {diode }}}{C_{\mathrm{Bl}}+C_{\text {diode }}} .
$$

\section{Conclusions and outlook}

We have proposed a type of resonator which has to our knowledge not yet been used for the purpose of resistive cooling. In order to be ensure that a resonant circuit created by a single crystal resonator is able to efficiently cool trapped particles further measurements are necessary. For the near future, measurements using a Penning trap with trapped electrons or protons are planned. If it turns out that the selectivity needs to be reduced in order to couple more modes of trapped particles the crystal's parallel resonance could be chirped over the limited range of accessible frequencies (see Figs. 10 and 11). Another option might be to incorporate a crystal bandstop filter instead of the single crystal resonator since the filter combines a high input impedance with a higher bandwidth. The bandstop filter allows to dynamically cover a frequency range comparable to that of parallel tuned circuit with a coil.

\section{References}

[1] Itano, W.M., Bergquist, J.C., Bollinger, J.J., Wineland, D.J., Cooling methods in ion traps, Phys. Scripta T59 (1995) 106.

[2] Ghosh, P.K., Ion traps. The international series of monographs on physics. Oxford: Clarendon Press, 1995.

[3] Sessler, A.M., Comment on the word "cooling" as it is used in beam physics, AIP Conf. Proc. 821 (2006) 3.

[4] Brown, L.S., Gabrielse, G., Geonium theory, Rev. Mod. Phys. 58 (1986) 233.

[5] Malmberg, J.H., O’Neil, T.M., Phys. Rev. Lett. 39 (1977) 1333.

[6] Dubin, D.H.E., Phys. Rev. Lett. 66 (1991) 2076.

[7] Wineland, D.J., Dehmelt, H.G., Principles of the stored ion calorimeter, J. Appl. Phys. 46 (1975) 919.

[8] Kracke, H., Entwicklung der kryogenen Nachweis-Elektronik zur Bestimmung der axialen Frequenz des Protons in einer Penning-Falle. Diploma thesis, University of Mainz, 2007. 
[9] Tietze, U., Schenk, C., Halbleiter-Schaltungstechnik - 12th ed., Berlin Heidelberg: Springer, 2002.

[10] A. Arnau, Piezoelectric transducers and applications - 2nd ed., Berlin : Springer, 2008.

[11] HC49-4H and HC49-4HL Crystals data sheet, Euro Quartz, 2010.

[12] HC49/U Standard Height Crystal data sheet, AEL Crystals, 2010.

[13] 404-29/41 data sheet, KVG Crystal Technology, 2010.

[14] Q-15-15-03-N data sheet, Webcraft, 2010.

[15] El Habti, A., Bastien, F., Low temperature limitation on the quality factor of quartz resonators. In: Proceedings of the 46th Annual Symposium on Frequency Control, 1992.

[16] Brendel, R., El Hassani, C., Influence of magnetic field on quartz crystal oscillators. In: Proceedings of the 43rd Annual Symposium on Frequency Control, 1989.

[17] Rychen, J., Ihn, T., Studerus, P., Herrmann, A., Ensslin, K., Hug, H.J., van Schendel, P.J.A., Guntherodt, H.J., Operation characteristics of piezoelectric quartz tuning forks in high magnetic fields at liquid helium temperatures, Review Sci. Instrum. 71 (2000) 1695. 